Sociologie et sociétés

\title{
La rationalité pénale moderne, la société du risque et la juridicisation de l'opinion publique
}

\section{Alvaro P. Pires}

Volume 33, numéro 1, printemps 2001

Les formes de la pénalité contemporaine : Enjeux sociaux et politiques

URI : https://id.erudit.org/iderudit/001562ar

DOI : https://doi.org/10.7202/001562ar

Aller au sommaire du numéro

\section{Éditeur(s)}

Les Presses de l'Université de Montréal

\section{ISSN}

0038-030X (imprimé)

1492-1375 (numérique)

Découvrir la revue

Citer cet article

Pires, A. P. (2001). La rationalité pénale moderne, la société du risque et la juridicisation de l'opinion publique. Sociologie et sociétés, 33(1), 179-204. https://doi.org/10.7202/001562ar d'utilisation que vous pouvez consulter en ligne. 


\section{La rationalité pénale moderne, la société du risque et la juridicisation de l'opinion publique ${ }^{\star}$}

ALVARO P. PIRES

Département de criminologie

Université d'Ottawa

25, rue Université

Ottawa (Ontario), Canada $\mathrm{K}_{1} \mathrm{~N} 6 \mathrm{~N}_{5}$

Courriel : alpires@uottawa.ca

C

et article Veut attiRer l'attention sur certaines caractéristiques de la rationalité pénale moderne et explorer quelques aspects de l'interaction entre les systèmes économique, politique et juridico-pénal dans les sociétés contemporaines (société du risque). Compte tenu de la complexité et de l'étendue des problèmes soulevés, je ne peux exposer ni toutes les principales conséquences qu’on peut en tirer ni développer les débats sur les points retenus. Ce texte suscitera aussi un ensemble de questions que je laisserai ici sans réponse ou qui n’apparaîtront que sous la forme de pistes éparpillées. Ainsi, le but premier de cet article n'est pas de présenter une analyse achevée de la situation contemporaine, mais plutôt de produire certains effets de miroir dans le double sens de faire apparaitre des choses absentes et de refléter notre propre image concernant notre manière de penser et de faire en matière pénale, y compris sur le plan de la recherche.

Sans doute la lacune principale face aux multiples interrogations que peut susciter cet article loge au niveau de l'élaboration de mon propre modèle normatif alternatif. On comprendra, par ailleurs, que je ne peux pas entreprendre les deux démarches en même temps et que la valeur éventuelle de l'une ne dépend pas rigoureusement de la qualité

* Je remercie Margarida Garcia, André Jodouin, Colette Parent, Gérald Pelletier et l'appréciateur anonyme de cet article pour leurs commentaires et suggestions. Ce travail s'inscrit dans le cadre d'un programme de recherche subventionné par le CRSH. Je remercie le Conseil pour l'aide accordée à ce projet. 
de l'autre : je peux décrire assez bien la situation actuelle et avoir quand même en tête un piètre modèle alternatif ou vice-versa. Ici, je dois espérer que les effets de miroir puissent avoir un certain intérêt et que la description (théorique) proposée soit au moins éclairante à certains égards. Par rapport au modèle normatif sous-jacent, peutêtre le seul éclaircissement à donner est le suivant. Ce modèle ne trouve pas ses assises dans la reconnaissance du pluralisme et il ne met pas en cause le principe de l'universalité de certaines lois en matière juridique ou pénale. Le pluralisme et une certaine universalité de normes sont paradoxalement deux réalités dont nous devons reconnaître simultanément l'existence. J'invite alors la lectrice ou le lecteur à noter que la critique qui se dégage de la description de la rationalité pénale ne met pas directement en cause l'universalité de certaines normes de comportement. Ce que cette description met essentiellement en cause, c'est notre façon de concevoir et de construire la solution institutionnelle à certains problèmes de transgression normative en matière pénale. Dans ce champ, les remarques de Carbonnier (1972, chap. II, section 2) ont une signification théorique capitale : il souligne que le véritable pluralisme juridique se trouve plutôt dans les diverses manières d'appliquer une norme (de comportement) que dans une franche opposition entre deux normes de comportement. D’ailleurs, en droit pénal, la portée critique de l'argument du pluralisme de normes de comportement est très limitée. Car on emploie cet argument à l'égard de certains crimes contre la moralité, la liberté d'expression religieuse, politique ou artistique, etc., mais rarement on y inclut le plus insignifiant des vols ou la plus anodine des incivilités comme faisant partie des normes de comportements qui doivent être honorées officiellement. La tolérance ici doit changer de niveau : elle continue à affirmer la norme qui interdit le comportement, mais propose une façon moins guerrière et «plus citoyenne» de s'adresser à la transgression. On peut même dire que la carence d'une pensée critique efficace en droit pénal résulte en partie du fait que nous ne nous sommes pas rendu compte que nous pouvons affirmer nos normes (universelles) de comportements de différentes façons, et non seulement par des peines afflictives (proportionnelles ou indéterminées). Nous n'avons pas appris à penser en termes d'alternatives. Dans cette étude, le lecteur trouvera sans doute un reproche de non-inventivité, mais il y a plus : il y trouvera aussi une inquiétude sérieuse à l'égard de la perte de qualité juridique du droit et à l'égard des formes «civilisées» d'avilissement qui ont été masquées par la rationalité pénale moderne. Or, l'universalisme de normes de comportement ne présuppose pas qu'il y ait une seule façon de faire les choses (uniformisation des pratiques) ou qu'elle soit dans tous les cas la meilleure ${ }^{1}$.

Dans un premier temps, je vais indiquer un aspect de la structure normative du droit pénal important pour mes propos et surtout souligner certaines caractéristiques de la rationalité pénale moderne en soutenant qu'elle se présente sous la forme d'un

1. Le lecteur se rendra compte aussi que l'autorisation juridique d'intervenir, de corriger des situations et même de punir n'est pas contestée. Par contre, la prétendue obligation de punir, les définitions de la punition, de la souffrance et de la justice données par le droit pénal - aussi bien que l'opérationnalisation de sa façon d'intervenir et de punir — sont effectivement mises en cause. 
«système de pensée» (Foucault) relativement autonome. Ceci permettra de mettre en lumière des problèmes fondamentaux que le droit pénal moderne pose - dès sa naissance aux XVIII ${ }^{\mathrm{e}}$ et XIX ${ }^{\mathrm{e}}$ siècles - aux sociétés démocratiques ${ }^{2}$. Par la suite, je me tournerai vers des transformations dans le système économique qui ont une influence sur le système pénal et qui suscitent de multiples formes d'auto-irritation dans ce dernier système. Ces transformations, en conjugaison avec les sciences sociales et les sélections opérées - et le style adopté - par le système pénal font éclater en morceaux et mettent en veilleuse les grands projets de mutation globale du droit pénal qui émergent dans les années 1960 et 1970. Enfin, dans un dernier temps, je reviendrai à la rationalité pénale moderne pour conceptualiser un phénomène nouveau : ce que j'appelle la juridicisation de l'opinion publique et du public par le système pénal dans la deuxième moitié du $\mathrm{xx}^{\mathrm{e}}$ siècle : le public devient une composante du système pénal et cela produit un rapprochement problématique avec le système politique et les projets de toutes sortes de mouvements sociaux en ce qui concerne la construction d'une notion autonome de justice en droit pénal, et particulièrement par rapport aux normes de deuxième niveau (sanction, procédure, etc.). Ajoutons encore que les rapports problématiques et contradictoires que le droit pénal entretient avec les droits de la personne traversent le texte d'un bout à l'autre. Très prosaïquement, on dira que le droit pénal est surtout vu comme une défense (négative) des droits de la personne mais que, à l'occasion et plus difficilement, les droits de la personne sont vus aussi comme un idéal pour transformer le droit pénal.

\section{ASPECTS de LA RATIONALITÉ PÉnALE MODERNE COMME SYSTÈme DE PENSÉE}

La rationalité pénale moderne est une manière de penser et de construire le droit pénal qui prendra une forme systémique autonome entre la seconde moitié du XVIII ${ }^{\mathrm{e}}$ siècle et le tournant du XIX ${ }^{\mathrm{e}}$ siècle (Pires, 1998a). Elle est un système social d'un genre particulier, c'est-à-dire un réseau de sens ayant une unité propre sur le plan du savoir et influant sur notre façon de construire, voir et justifier une institution spécifique, en l'occurrence le droit pénal moderne. Elle possède la capacité de naturaliser la structure normative des lois pénales et ses pratiques institutionnelles. C'est quand nous essayons de penser le système pénal autrement que nous prenons conscience de l'emprise de ce système sur notre façon de voir les choses.

Ce système de pensée se met en place dans le cadre d'un processus où le droit se différencie à l'intérieur du droit, c'est-à-dire d'un processus de création de divers sous-systèmes de droit. Le droit pénal moderne va se construire alors — et sera perçu — comme un sous-système relativement autonome et différencié des autres sous-systèmes juridiques. Ceci lui donnera une pesanteur sociologique et une énorme force d'attraction culturelle dans le contexte d'une modification du droit, soit dans le traitement de

2. C'est ici que nous pouvons observer certaines déficiences morales «innées» significatives de cette rationalité. 


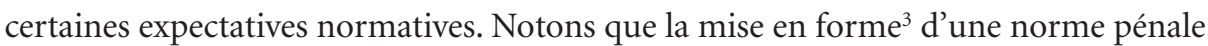
interdisant ou prescrivant un comportement va prendre, usuellement, une structure télescopée du genre suivant : «celui qui fait $\mathrm{x}$ doit être puni par $\mathrm{y}$ ». Elle est télescopée parce qu'elle juxtapose une norme de comportement (ne pas faire ceci ou faire obligatoirement cela) et une norme sanctionnatrice ou d'application du droit (permettant ou obligeant l'application de la sanction désignée). Cette mise en forme est alors le résultat d'un carambolage (ou de l'accouplement) de deux niveaux distincts de normes : des normes du premier degré (comportements) et du second degré (normes de sanction $)^{4}$. Qui plus est, cette structure télescopée accordera une visibilité particulière à trois types de peines (normes du deuxième degré) : la mort (ou un autre châtiment corporel), la prison et l'amende. Et c'est la peine afflictive qui prend une place dominante, et tout particulièrement la prison.

Parallèlement à la mise en place de cette structure, on rendra officielle une ligne de pensée médiévale selon laquelle c'est la peine afflictive qui communique la valeur de la norme de comportement et le degré de réprobation en cas de non respect. La quantité du mal (norme de sanction) communiquerait le degré d'attachement au bien (norme de comportement honorée). En effet, à partir du moyen-âge, et relié au processus d'affirmation du pouvoir du Prince, on commence à stigmatiser comme un trait de la culture barbare ou d'une civilisation peu évoluée le fait de réagir juridiquement à la transgression de certaines valeurs par des sanctions de dédommagement ou, plus globalement, par des sanctions non afflictives ${ }^{5}$. Les sciences sociales, pendant leur période de formation au cours du XIX ${ }^{\mathrm{e}}$ siècle, vont aussi reconduire et valider cette représentation. On la retrouve (entre autres) chez Durkheim (1969, p. 143) lorsqu'il traite des normes relatives à l'homicide : «Ce qui prouve la moindre dignité morale qui leur était alors attribuée, c'est la moindre gravité des peines qui les sanctionnaient. [...] En Grèce, le meurtre luimême n'était puni que sur la demande de la famille et celle-ci pouvait se contenter d'une

3. Le concept de «mise en forme pénale» a été proposé par Acosta (1987, p. 2) pour désigner le processus de construction de la transgression pénale à travers la transformation progressive de ce qui était au départ une trame de vie (c'est-à-dire un ensemble diffus d'événements) en fait juridique (crime). L'enquête (policière, judiciaire) est le pivot de ce processus. J'adapte ce concept pour désigner aussi la fabrication de la structure normative des normes pénales elles-mêmes.

4. Nous nous inspirons ici de la définition de règles primaires et secondaires proposée par Hart (1961) avec les modifications de Gavazzi (1967). Pour Hart, les normes primaires sont celles qui prescrivent à des individus d'accomplir ou de s'abstenir de certains comportements. Les normes secondaires, par contre, sont "parasitaires par rapport aux normes primaires» (p. 105); elles occupent un autre niveau (Gavazzi, 1967, p. 146-147) : elles sont des normes qui se rapportent aux normes primaires (Hart, 1961, p. 119); des normes sous des normes. C'est Gavazzi qui recommande d'abandonner la terminologie primaire/secondaire pour la remplacer par «règles du premier et du deuxième degré».

5. Marsilio de Padua (1275-1343) écrit dans un ouvrage publié en 1505: «Un exemple [d'une loi imparfaite] peut être trouvé dans certains pays barbares, où on tient pour une norme juste le fait d'être disculpé et exonéré de toute punition civile [de la cité] d'un homicide, pourvu qu'il paie un certain montant pour tel délit» (dans Il defensore della pace, 1960, \$5, p. 156, cité par Bobbio, 1961, p. 141). Notons que cet auteur soutient aussi la thèse politique de l'autonomie complète de l'État par rapport au droit canon et à l'Église, y compris par rapport au droit naturel. 
indemnité pécuniaire» (mon souligné). Durkheim corrigera cette interprétation dans d'autres textes, mais ils ne sont pas d'ordinaire repris dans la question pénale.

Cette combinaison de facteurs (structure télescopée et peine afflictive valorisée) donnera l'impression que crime et peine forment un tout inséparable, suscitant une série de problèmes, glissements de sens et de confusions. Indiquons-en trois. D’abord, cette combinaison donnera une formidable illusion de nécessité et d'identité quant à la nature de cette association entre crime et peine (afflictive). On suppose que les normes de comportement et de sanction sont (ou doivent être) de la même nature, et ce en deux sens différents : (a) les deux normes relèveraient de la même sorte de devoir être, c'est-à-dire seraient également obligatoires ${ }^{7}$ et (b) la sanction pénale devrait, comme le crime, découler d'un acte de volonté qui cherche directement et intentionnellement à produire le mal (souffrance). Ensuite, cela donnera aussi une illusion de simplicité quant à la détermination de la sanction pénale : elle paraît aller de soi et devoir privilégier d'emblée l'incarcération ou la peine afflictive. Enfin, divers discours savants vont dogmatiser immédiatement la relation crime/peine (afflictive) : "pas de crime sans peine» (nullum crimen sine poena legali; Feuerbach, 1801, p. 41). Et sur le plan de la réflexion théorique, autant le droit que les sciences sociales vont définir le crime (ou le système pénal) par la peine (afflictive) ${ }^{8}$ et s'engagent dans une compréhension simultanée des deux types de normes. Ces savoirs donnent alors une définition de leur objet qui peut être qualifiée de «synedocquetique» pour reprendre cette figure de rhétorique qui consiste à prendre le plus pour le moins (ex. les mortels pour les hommes; le système pénal pour la peine), etc. Cette erreur rend quasi impossible la tâche de penser le système pénal (ou le crime) sans un attachement exclusif à la peine afflictive et ouvre grandes les portes à une ontologisation de la structure normative du droit pénal.

Par conséquent, il arrive à l'égard de l'étude du système pénal et du crime quelque chose de semblable à ce que Bourdieu (1993) identifie vis-à-vis l'étude de l'État («Esprits

6. Voir, par exemple, le débat de Durkheim (1895) avec Tarde sur la «Société des saints» où le premier finit par distinguer le degré de réprobation du degré de répression. Et voir, tout particulièrement, les trois importants chapitres de Durkheim (1963) sur la pénalité scolaire dans L'éducation morale.

7. Comme le note Kelsen (1979, p. 3), toute norme statue un devoir-être, «mais ce mot englobe toutes les fonctions normatives possibles : commander, habiliter, permettre, abroger ». Si l'on tient compte des deux niveaux de norme (premier et deuxième degré), il est alors possible d'introduire ou de distinguer deux fonctions normatives dans la mise en forme de la norme pénale : «Quiconque fait ou ne fait pas X» (fonction de commandement obligatoire) "peut ou non être sanctionné et, si sanctionné, de telle ou telle façon» (fonction d'autorisation adressée surtout aux autorités). Dès lors, dans le langage kantien, un devoir-être exprimant un impératif catégorique (norme du premier degré) peut coexister parfaitement avec un devoir-être exprimant un impératif hypothétique «comme moyen pour quelque autre chose» (Kant, 1785, p. 125), soit comme norme permissive du deuxième degré pour réaffirmer au besoin la norme du premier degré.

8. Ces définitions diront, par exemple, que «le système pénal est le sous-système de droit qui applique des peines» ou que «le crime est toute conduite que le droit criminel incrimine par la menace d'une peine». Moi-même, j'ai donné ce genre de définition dans le passé. Elles sont relativement acceptables d'un point de vue empirique, mais nous faisons l'économie d'une bonne définition théorique : qu'est-ce qui arrive si un comportement devient incriminé par la menace d'un dédommagement? Le système pénal, ou le crime, disparaît-il? Voir Pires (2001b). 
d'État») : les sciences sociales, la philosophie et le savoir juridique éprouvent de grandes difficultés à penser le crime et le système pénal sans appliquer à ces objets les catégories de pensée produites et garanties par la rationalité pénale elle-même. L'esprit de peine obnubile la pensée des chercheurs qui étudient le système pénal ou le droit pénal. En effet, ces savoirs ne réussissent pas à prendre suffisamment de recul par rapport au système de pensée et aux pratiques institutionnelles qu'ils sont censés décrire et analyser. Cette distance critique exige la possibilité théorique de pouvoir décrire la configuration effective de ce système comme étant une possibilité parmi d'autres (et non nécessairement la plus heureuse) d'actualisation du système. La rationalité pénale moderne constitue alors un obstacle épistémologique à la connaissance de la question pénale et, en même temps, à l'innovation, c'est-à-dire à la création d'une nouvelle rationalité et d'une autre structure normative. Voici quelques aspects de ce système de pensée.

1. Tout d'abord, à partir du XviII ${ }^{\mathrm{e}}$ siècle, le droit pénal va s'auto-observer et projeter un auto-portrait essentiellement punitif de lui-même. À ses yeux, seulement une procédure hostile et autoritaire - qui empêche la victime de résoudre autrement le conflit - et accompagnée de sanctions afflictives peuvent nous défendre vis-à-vis le crime (Pires, 2001a). Une particularité du droit pénal est qu'il va créer et stabiliser une double expectative normative : la première porte sur le comportement et l'autre sur la sanction afflictive. On attend alors aussi telle souffrance comme réponse supposée juste, proportionnelle ou nécessaire à la transgression. Cela donne la fausse impression que le droit pénal a pour fonction essentielle — voire comme unique fonction d'appliquer la même peine afflictive (égalité de nature et d'effets) à tous ceux qui commettent le même type de transgression.

2. Le foyer dominant de cet auto-portrait punitif est composé essentiellement des théories classiques de la peine (de la dissuasion et de la rétribution), ou encore de certaines variantes contemporaines qui excluent les formes «positives» ou alternatives de résolution des conflits. Ces théories classiques vont concevoir la protection de la société (ou l'affirmation des normes) de façon hostile, abstraite, négative et atomiste. Hostile, parce qu'on représente le déviant comme un ennemi du groupe tout entier et parce qu'on veut établir une sorte d'équivalence nécessaire, voire ontologique, entre la valeur du bien offensé et l'affliction à produire chez le déviant. Abstraite, parce que le mal (concret) causé par la peine est reconnu mais conçu comme devant causer un bien moral immatériel («rétablir la justice par la souffrance», «renforcer la moralité des gens honnêtes», etc.) ou encore un bien pratique invisible et futur (la dissuasion). Négative puisque ces théories excluent toute autre sanction visant à réaffirmer le droit par une action positive (le dédommagement, etc.) et stipulent que seul le mal concret et immédiat causé au déviant peut produire un bien-être pour le groupe ou réaffirmer la valeur de la norme. Et, enfin, atomiste, parce que la peine - dans la meilleure des hypothèses — n'a pas à se préoccuper des liens sociaux concrets entre les personnes sauf d'une façon tout à fait secondaire et accessoire. Ces théories généralisent à l'ensemble du droit pénal cette «justification quasi médicale», comme l'appelle Luhmann (1997, p. 283), que l'on trouve déjà joliment formulée dans un texte de Pierre Ayreault 
de 1576 portant sur l'ordre et la formalité dans l'instruction judiciaire : «le mal se guarist par le mal».

3. Les théories classiques de la peine ont fondé le droit de punir aux XVIII ${ }^{\mathrm{e}}$ et XIX siècles comme une obligation (ou nécessité) de punir plutôt que comme une simple autorisation de punir. Elles vont alors mettre en échec le principe juridique médiéval de l'intervention en dernier ressort (ultima ratio) repris à d'autres égards par la pensée libérale à partir du $\mathrm{XvII}^{\mathrm{e}}$ siècle. Ainsi, dans le cadre de la théorie de la dissuasion, Beccaria affirme, par exemple, que la certitude de la peine est plus importante que sa sévérité. Ce propos est souvent présenté comme s'il était critique, mais il reconduit par là l'idée qu'il existerait une sorte d'obligation pragmatique et politique de punir. Kant affirme, quant à lui, dans le cadre d'une théorie de la rétribution, que la peine est un impératif catégorique (obligation morale de punir) ${ }^{9}$. Dans le fondement moderne du droit de punir s'opère alors un glissement de sens allant de l'autorisation (droit-faculté) à l'obligation de punir (droit-obligation). Ceci est facilité par le fait que les mots droit et obligation sont asymétriques : qui $a$ un droit n'est pas nécessairement obligé de faire quelque chose, mais qui est obligé a nécessairement le droit de le faire (Pires, 1998c).

Certes, il est difficile de savoir quand la croyance en une obligation de punir prend une forme systémique et devient dominante. L'hypothèse que je fais, c'est que cela ne se produit qu'à partir du XviII ${ }^{\mathrm{e}}$ siècle. Quoi qu'il en soit, il est intéressant de voir que ce point de vue contraste avec celui de Grotius dans le premier quart du XviI siècle. Grotius (1625) donne aux notions de guerre, crime et punition un sens très large et soutient que seule la guerre défensive ou rétributive est autorisée, c'est-à-dire la guerre comme légitime défense ou punition (rétribution). En ce sens, la punition doit, par définition, être rétributive (malum passionis propter malum actionis). Mais le rétributivisme, pour lui, ne se confond ni avec l'obligation de punir ni avec les seules sanctions du droit pénal moderne (mort, prison, amende). En effet, il écarte toute ambiguïté autour du terme autorisation : la guerre (ou punition) rétributive n'est jamais obligatoire. Le droit de punir et le droit de la guerre ont la même source : «il naît du crime du coupable». Mais, insiste-t-il, il ne faut surtout pas confondre cet acte de naissance avec une quelconque obligation de punir. Le crime rend l'individu (ou l'État) coupable apte à la peine, mais ce n'est que par un abus du langage qu'on dit que la peine «est due au délinquant». En effet, pour Grotius, ceci serait un glissement de sens et un jeu du langage car la formule rétributiviste la peine est due au crime signifie essentiellement la peine doit sa naissance au crime et non la peine est due au délinquant au sens de devoir être infligée. Le droit-faculté de punir exige que l'individu soit en situation de pouvoir mériter la punition, mais de même que pour la récompense, cela ne signifie pas qu'elle soit due au

9. Littéralement, Kant écrit que la «loi pénale» (Das Strafgesetz), et non la peine, est un impératif catégorique. Cependant, par le contexte, le renvoi à la loi du Talion et les exemples qu'il donne, il est évident qu'il pense même plus à la peine qu'à la norme de comportement. Ceci est un bon exemple du carambolage entre les deux niveaux de normes et la tendance à glisser de l'«obligatoriété» de la norme de comportement à la supposée «obligatoriété» d'une peine afflictive proportionnelle (Pires, 1998b, p. 179) Voir aussi les remarques de Muller (1995, p. 145-152; 184) sur le mimétisme de la violence du système pénal. 
sens propre d'une obligation. L'obligation d'obéir à la norme de comportement n'est pas attachée à une obligation d'infliger une sanction, et moins encore une sanction afflictive, puisqu'il y a d'autres manières d'affirmer la norme. Grotius, qui réfléchit ici exclusivement sur les conditions pour donner une sanction (et non sur la façon de le faire), ajoute que l'on ne fait pas d'injustice aux coupables s'ils sont punis, «mais cela ne signifie pas que tous doivent être absolument punis $»^{10}$.

4. Enfin, en transposant une expression de Feeley et Simon (1994, p. 190), on peut dire que la rationalité pénale moderne est un système de pensée "profondément prépolitique» ou trans-politique : il se sédimente avant ou indépendamment des visions politiques du monde. Ce sous-système culturel est alors très puissant puisqu'il n'est pas nécessairement interrogé ou problématisé par les diverses orientations politiques (Pires, Cellard et Pelletier, 2001). On peut dire que les notions politiques de gauche et de droite ne produisent pas de différences cohérentes et remarquables en matière pénale, car nombre de membres de l'inteligencija (Boborykin), de gauche ou de droite, croient en l'une ou l'autre des théories officielles de la peine. Une des conséquences de cela est que des individus, groupes et mouvements sociaux qui ne sont pas à proprement parler conservateurs peuvent alors, sans contradiction à leurs yeux, demander une plus grande sévérité des peines ou s'opposer à tout adoucissement de peines, voire aux sanctions alternatives.

Avant d'examiner quelques transformations dans les sociétés contemporaines, faisons une brève parenthèse pour rappeler que dans les années 1960 et 1970 le savoir juridique et les sciences sociales se trouvaient dans une période d'auto-réflexion critique par rapport au droit pénal. Pendant cette période, qui coïncide avec le moment où des transformations sociétales importantes s'installent et se préparent pour prendre un rythme plus accéléré, on voulait construire une nouvelle rationalité pénale et revoir entièrement la structure normative du droit pénal. On peut dire, en employant les termes de Bloch (1961), que le droit pénal construisait alors pour lui-même une utopie juridique, c'est-à-dire une auto-réflexion consistant «dans le dépassement du donné, dans la foi que ce qui est présent doit être supprimé pour ouvrir la voie à un statut meilleur» (p. 210). Ce statut meilleur consisterait en une démarche d'auto-perfectionnement pour mieux rendre compte de la dignité humaine dans ses principes d'intervention et d'opération au sens large.

Concrètement, au Canada, les commissions de réforme du droit de cette époque voulaient transformer les théories de la peine, diversifier les formes de procédure pénale pour faciliter l'entente entre les parties et la déjudiciarisation, réduire la durée légale et effective des peines d'incarcération, transformer l'emprisonnement dans une peine réellement exceptionnelle par l'adoption d'autres remèdes ou sanctions juridiques, éli-

10. Comme Grotius traite en même temps du droit pénal et du droit international, il est plus facile pour lui d'écarter certaines absurdités. C'est que la variante de la théorie rétributiviste adoptée par le droit pénal moderne, appliquée au droit international, aurait stimulé la guerre et, de nos jours, aurait détruit la planète. Del Vecchio (1955) adopte, à cet égard, une position voisine à celle de Grotius, mais elle sera aussi marginalisée par le droit pénal moderne. 
miner toutes les peines à perpétuité aussi bien que les peines minimales d'incarcération, etc. Les commissions voulaient aussi clarifier les critères de jugement (notion de responsabilité et de gravité) et, si possible, réduire le nombre de lois pénales de comportement ${ }^{11}$. L'idée de justice réparatrice, les mouvements «au-delà du droit» (beyond law) ou pour l'abolition du système pénal, etc., plongent ses racines dans cette période de réflexion et d'auto-réflexion critique en matière pénale.

De façon plus remarquable, on essayait de construire une nouvelle image du droit pénal et de modifier l'orientation de ses rapports avec les droits de la personne. Dans le système juridique, le droit pénal avait (et a encore) une image qui rappelle celle du droit procédural. En effet, il est vu comme une sorte de «droit de deuxième ordre», d'un droit (plus vigoureux) de protection du droit, dont l'objectif premier serait de protéger le droit tout entier contre certaines attaques désignées, contribuant ainsi à maintenir l'ordre mais sans contribuer directement et positivement à la configuration de la paix sociale et des droits eux-mêmes ${ }^{12}$. Cette image du droit pénal crée une sorte de paradoxe face aux droits de la personne. Car le premier sera représenté d'ordinaire comme un droit de protection (négative) du second. Et il ne doit tenir compte des droits de la personne que sur le plan de sa légalité et de certains principes généraux de sa procédure. Tout se passe comme s'il ne pouvait pas à la fois défendre les droits de la personne et exprimer concrètement et immédiatement ces droits contribuant à sa configuration ${ }^{13}$. En ce sens, les droits de la personne sont en partie neutralisés à titre de principes de réforme humaniste du droit pénal lui-même, car c'est à ce dernier que revient la fonction de protéger les droits de la personne et non aux droits de la personne d' «affaiblir» leur propre protection en humanisant le droit pénal. L'humanisation du droit pénal est représentée alors comme un signe de faiblesse, de mollesse, au plus d'indulgence, mais pas de justice.

Selon Honneth (1999, p. 11-12), la première prémisse de Bloch est que la «dignité humaine ne peut se révéler que de manière indirecte : en identifiant les formes d'avilissement et d'offense infligées à la personne»; la deuxième est que "ce sont seulement de telles expériences négatives du mépris et de l'outrage qui peuvent donner une impulsion pratique à une orientation normative visant à assurer la dignité humaine». Si ces prémisses de Bloch sont fondées, on peut comprendre un peu mieux pourquoi nous éprouvons tant de difficultés à identifier les formes d'avilissement mises en œuvre par la rationalité pénale et par le droit pénal : c'est qu'il s'agit de formes «civilisées» d'avilissement et elles sont vues comme nous protégeant de l'avilissement. L'expérience du mépris et de l'outrage mis en œuvre par le droit pénal se transforme immédiatement en une expérience positive de soulagement et en une conceptualisation de la justice. Or,

11. On peut se demander si ce dernier objectif n'est pas entièrement périmé face à la complexification croissante de la vie sociale. Les autres, par contre, demeurent accessibles car ils impliquent ce qui est à la portée du système pénal lui-même : une complexification et un ajustement internes pour s'adapter à la complexité de son environnement.

12. Cette représentation du droit pénal est présente aussi dans la description donnée par la sociologie du droit. Voir, par exemple, Ehrlich (1986, p. 99).

13. Voir les réflexions classiques de Mead (1918). 
c'est justement cette image qui faisait objet d'examen dans les années 1960 et 1970 lorsque la société du risque entre, selon le schéma de Beck, dans le deuxième stade de son évolution et que d'autres transformations inattendues se succèdent. Celles-ci contribueront à l'auto-irritation du système pénal et des sciences sociales. Du coup, les projets d'une réforme de fond et d'ensemble donnent lieu à une bifurcation incertaine : ces projets ne disparaissent pas, mais ils sont contrecarrés par une relance de la rationalité pénale moderne. L’utopie juridico-pénale comme telle est renvoyée aux calendes grecques.

\section{LA SOCIÉTÉ DU RISQUE OU LE NOUVEL ENVIRONNEMENT SOCIAL DU SYSTÈME PÉNAL}

À partir d'ici, je mets surtout en place quelques hypothèses d'un travail en cours. Prenons comme point de départ le fait que l'on reconnaît aujourd'hui en sociologie que les sociétés occidentales avancées ont subi des transformations structurelles importantes pendant la deuxième moitié du $\mathrm{xx}^{\mathrm{e}}$ siècle. Je veux explorer librement ici ce que Beck $(1986 ; 1993 ; 1994)$ appelle l'émergence de la «société du risque» ${ }^{14}$. Le foyer de cette transformation est le système économique ou le marché.

Beck $(1986$; 1994) a employé la notion de société du risque pour désigner «une phase de l'évolution de la société moderne dans laquelle les risques sociaux, politiques, écologiques et individuels engendrés par la dynamique du renouvellement, se soustraient de plus en plus aux instances de contrôle et de sécurité de la société industrielle». La société du risque s'oppose alors à la société industrielle. Selon Beck (1994, p. 333), la mise en place de la société du risque passe par deux stades. Pendant le premier, caractérisé par la mise en place du complexe industriel-militaire après les années 1950, des nouveaux dangers industriels «sont créés de manière systémique, mais ne font pas l'objet d'une thématisation publique ni ne se situent au centre de conflits politiques». C'est la philosophie de la société industrielle qui prédomine et celle-ci a tendance à minimiser les risques et à les présenter comme étant résiduels (ibid.). La situation se modifie qualitativement au deuxième stade, car alors «la société industrielle se considère comme société du risque et se critique comme telle» (ibid.). Beck (1986, p. 20) fixe celui-ci — au moins par rapport à l'Allemagne — à partir des années 1970.

Je dirais que la notion de société du risque désigne la conjonction de différents aspects des sociétés contemporaines. Tout d'abord, une nouvelle rationalité dominante est mise en place pendant la période de la société industrielle. Cette rationalité est très importante mais je ne peux pas traiter ce point ici. Ensuite, le fait que la société, à partir d'un moment donné, commence à produire des nouveaux «dangers décidés» de nature industrielle. Puis le fait que ces dangers échappent de plus en plus aux formes de contrôle institutionnel, voire de réparation, et deviennent plus visibles socialement.

14. La traduction française de l'expression allemande Risikogesellschaft est encore incertaine. Elle prend tantôt la forme société à risques, tantôt société de risques, tantôt encore société du risque. Nous privilégions cette dernière. 
Enfin, des dangers socio-économiques reliés à la mondialisation viennent s'ajouter aux dangers industriels, même si Beck met l'accent sur les derniers.

De la même façon que pour Luhmann $(1979$; 1993) et Giddens (1990), le concept de risque est construit autour de la décision. Le mot «risque» naît dans la modernité «avec la conscience que les résultats non anticipés [et indésirables] peuvent provenir de nos propres activités ou décisions» (Giddens, 1990, p. 30). Dès lors, pour Beck (1994), la société industrielle assume des risques et essaye de maitriser ou de compenser les dangers d'une quelconque manière (par des calculs de risque, des systèmes d'assurance, etc.).

\section{1) Les dangers industriels et la rationalité pénale moderne}

Selon Beck, les nouveaux dangers industriels (surtout les dangers nucléaires, chimiques, écologiques ou reliés à la technologie génétique, etc.) sont qualitativement différents et se distinguent progressivement des anciens dangers par quatre caractéristiques. Tout d'abord, en raison de leurs causes. Ils résultent plutôt d'une superproduction que d'une insuffisance de technologie ou d'hygiène. Et ils relèvent de décisions systémiques industrielles et scientifiques, "c'est-à-dire de choix arrêtés au vu d'avantages et de possibilités technico-économiques» (Beck, 1993, p. 312). Le nouveau danger «diffère des catastrophes naturelles de l'époque préindustrielle par cette origine décisionnelle», par le fait d'être auto-généré par l'industrie. En plus, certains de ces risques sont produits dans des circonstances de normalité et de légalité; d'autres découlent de la production d'armes de destruction massive et sont conditionnés en ce qui concerne leur mise en application (et non en ce qui touche leur potentiel de menace) à des situations exceptionnelles comme la guerre (Beck, 1997, p. 67-69). Il remarque aussi qu'il existe des dommages écologiques qui sont conditionnés à la fois par l'inégalité sociale et les dangers techno-industriels, comme la déforestation des forêts tropicales. Deuxièmement, les risques possèdent une portée globale et sont illimités dans le temps et dans l'espace. Troisièmement, ils «ne peuvent pas être imputés à quelqu'un ou à quelque chose selon les règles actuelles de la causalité, de la culpabilité, de la responsabilité». Enfin, ils «ne peuvent pas être compensés ni assurés» (Beck, 1994, p. 336). Bien entendu, le discours politique et scientifique sur le risque exclut à la fois le système économique et celui qui prédit le risque comme producteur du danger (par ses propres décisions). Comme il n'y a pas de décision sans risque, celui qui prédit n'est pas un simple acteur scientifique, mais aussi un acteur politique. Pour explorer ce problème, je ferai état de trois cas sans aucun souci de représentativité.

a. Trois chercheurs de l'Université de Toronto (Lazarou, Pomeranz, Corey, 1998) ont estimé que des médicaments correctement prescrits tuaient en moyenne cent mille personnes par année en Amérique du Nord. Même si pour des raisons méthodologiques on venait à réduire ce chiffre de la moitié, il n'en illustre pas moins les énormes pertes humaines qui échappent à tout système de contrôle, y compris juridique. Puis cet exemple montre aussi une facette du problème concernant le raisonnement de cause et effet qui est relié à la notion de responsabilité en droit pénal. En effet, il est quasiment 
impossible de remonter la chaîne causale vers l'individu coupable. En plus, il est étonnant de constater que l'acteur social le plus proche de l'effet, le médecin, est un acteur non responsable d'un danger qu'il transmet pourtant activement.

b. Beck (1993, p. 317-318) raconte qu'en République fédérale d'Allemagne, en 1985, des 13000 affaires de délits écologiques «instruites par les tribunaux, 27 ont abouti à un verdict d'emprisonnement, assorti de sursis dans 24 cas, toutes les autres affaires étant classées $»^{15}$. À son avis, une multitude de facteurs expliquent cela, allant des «lacunes de la loi et des carences notoires de son application», jusqu'à une application à la lettre du principe de causalité au plan de l'individu (Beck, 1993, p. 317). On peut aussi y voir en opération, me semble-t-il, un mode de raisonnement juridique distinct de celui de la rationalité pénale moderne. Par exemple, dans le cas d'une usine de cristal, dont la pollution avait produit des migraines, des nausées et des éruptions cutanées dans la population en plus d'avoir bruni les branches des arbres, rouillé les fenêtres, etc., le président du tribunal propose au dixième jour de procès l'abandon de la poursuite contre le paiement d'une amende de 10 ooo marks allemands. Beck (1993, p. 317-318) souligne que la responsabilité était indéniable, mais l'entreprise a bénéficié d'une circonstance atténuante ${ }^{16}:$ il y avait dans son voisinage trois autres verreries qui produisaient les mêmes saletés. Beck fait probablement une référence implicite au fait que le tribunal aurait supposé l'existence d'une causalité cumulative, quatre verreries contribuant au même effet. Il conclut : «plus on pollue, moins c'est pollué» (Beck, 1993, p. 318). Le lecteur peut imaginer aisément comment une circonstance atténuante de ce genre serait difficilement reçue en droit pénal ordinaire.

c. L'affaire du sang contaminé qui compte nombre de victimes en France et au Canada constitue un autre exemple. Contrairement à la France, le gouvernement et la justice au Canada ont décidé de ne pas entreprendre de poursuites criminelles. Selon les renseignements de la presse, le gouvernement aurait aussi exercé des pressions pour empêcher une commission d'enquête d'énoncer des blâmes individualisés. La résolution du conflit fut finalement politique : une indemnisation a été versée aux victimes ou aux familles des victimes déjà décédées. Il ne s'agit pas de déplorer la non intervention du droit pénal, mais plutôt de montrer qu'existent d'autres systèmes de régulation possible pour des actes qui sont susceptibles de criminalisation.

Des événements de cette nature peuvent, d'une part, auto-stimuler la rationalité pénale moderne qui relance les opérations usuelles du système. Par exemple, on peut vouloir créer des nouvelles lois pénales avec des peines terribles dans l'espoir de mettre en prison l'élite déviante. Mais, d'autre part, ces événements peuvent produire aussi un effet de mise en cause externe et interne au système pénal. Pour apprécier cet effet,

15. L'exemple de Beck peut créer une ambiguïté quant à la croyance en la prison pour résoudre ces problèmes. Quant à moi, ce que je veux souligner par là c'est le caractère inopérant des régulations actuelles et la futilité de vouloir régler ces problèmes dans le cadre de la structure normative actuelle du droit pénal et de son système de pensée.

16. Les termes de Beck (1993, p. 318) sont «une circonstance disculpante». En fait, ce que nous voyons en œuvre ici relève d'un mécanisme juridique de dilution de l'imputabilité. Je suis redevable à mon collègue Fernando Acosta pour cette observation. 
tournons-nous vers un projet de loi récent au Canada. Le ministère de la Justice a proposé que l'on porte à cinq ans la peine maximale pour le traitement choquant d'animaux familiers et il n'a fixé dans la loi aucune limite aux amendes pouvant être infligées. Selon les documents officiels, le gouvernement s'appuie sur des «études policières» pour soutenir que ces actes sont reliés «à la colère, au pouvoir et au contrôle». La proposition fait aussi une association entre ces comportements, la violence familiale et le meurtre en série (sic). Et on ajoute que les Canadiens ne peuvent plus tolérer ce genre de mépris à l'égard des animaux : «ils s'attendent à ce que le système de justice criminelle traite un tel comportement avec toute la rigueur possible». Étant donné, nous dit-on, que la rigueur actuelle de la loi n'exprime plus adéquatement nos valeurs et notre désapprobation, ne dissuade pas assez les gens et ne permet pas de «réadapter les délinquants violents», eh bien, il faut punir plus ${ }^{17}$. Bref, pour montrer que nous aimons plus nos animaux, nous devons souhaiter une peine de cinq ans à l'égard de notre co-citoyen qui les maltraite. On voit bien en ouvre la rationalité pénale : elle crée un lien pathologique entre les valeurs (amour, etc.) et la disposition à faire souffrir pour l'exprimer. Bien entendu, la loi ne fait rien pour améliorer directement la qualité de vie des animaux. Nombre de parcs publics sont interdits aux chiens, même attachés à une laisse ultra-sécuritaire. Et les directeurs de ces parcs ont droit à l'immunité...

Cette comparaison illustre un phénomène contemporain qui n'est pas spécifiquement canadien : l'émergence d'un mécanisme social externe-interne d'«auto-ridicularisation» du système pénal par la confrontation entre ce qui se passe d'important en dehors de son emprise avec ce que la rationalité pénale prend en charge et surtout la façon dont elle le fait. En m'inspirant de Beck, on peut parler ici de la naissance d'une critique sans sujet et sans discours. L'auto-ridicularisation du système pénal s'accomplit par elle-même. Bien sûr, l'observateur doit quand même prendre un peu de recul pour comparer deux types d'événements médiatisés : un portant sur l'aggravation de peines dans le Code criminel (par exemple, pour exprimer nos valeurs à l'égard des animaux) et un autre faisant état d'un cas semblable aux exemples ci-haut. Or, comme le système pénal a le contrôle sur sa propre légitimation, d'où vient cette nouvelle source de délégitimation du système? Paradoxalement, elle est rendue possible par l'interaction des transformations de la société avec les caractéristiques désuètes du système pénal : sa rationalité et la logique de sa structure normative («celui qui fait x doit être puni par

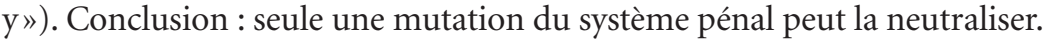

La deuxième modernité met en cause et irrite aussi le paradigme conventionnel de la menace qu'entretient l'auto-portrait du droit pénal. Pour celui-ci, au moins depuis le XVIII ${ }^{e}$ siècle, deux cas de figure ont constitué le prototype de ce qui mettait en cause les conditions de vie en société : l'homicide intentionnel et méchant d'un individu par un autre et le vol dans ses formes conventionnelles. Voilà ce qui menaçait l'existence de la société et qui rendait nécessaire des peines afflictives très sévères. Or, il est évident au-

17. Les passages entre guillemets sont tirés d'une fiche documentaire du ministère de la Justice et d'un document de consultation. Ces textes sont disponibles sur Internet (http://canada.justice.gc.ca/fr/nouv/ cp/1999/doc_24311.html). 
jourd'hui que non seulement la société est hautement résistante à ces méfaits mais aussi que sa destruction, si l'on veut parler dans ces termes, passe par d'autres voies plus plausibles (les nouveaux dangers décidés). Ces cas de figure éclairent aussi le sens donné à la notion de gravité. En effet, le savoir pénal classique met l'accent sur la faute individuelle détachée de ses racines sociales, soit sur l'action de cause à effet pleinement intentionnelle et mal intentionnée. Le pôle privilégié de la notion de gravité repose davantage sur la caractérisation et la qualification de l'intention que sur la portée et les conséquences de la décision. La lecture déterministe du Xix ${ }^{e}$ siècle, axée sur la dangerosité du délinquant, ne change pas radicalement ce tableau, car elle conserve l'individu comme pôle central de la détermination de la gravité. La deuxième modernité fauche l'herbe sous le pied de ce système de pensée. D'une part, elle enlève le caractère vraisemblable du paradigme conventionnel de la défense de la société et, du coup, rend visible l'excès de violence contenue dans sa réponse. Car plus les décisions ont des conséquences réellement fâcheuses, plus elles échappent à la mauvaise humeur du droit pénal. D’autre part, la notion de gravité devient confuse et incohérente, car le système réagit à ce qui se passe dans son environnement en valorisant de plus en plus le simple résultat de l'action et les risques de toutes sortes, ou encore en plaçant sur un pied d'égalité l'intention méchante et non méchante. Ceci crée une forme latente et interne de délégitimation cognitive, en plus d'une sorte d'imprévisibilité interne systémique.

\section{2) Les dangers décidés socio-économiques et la rationalité pénale moderne}

Par ses décisions systémiques socio-économiques, la société produit aussi des risques sur le plan des rapports sociaux. Les effets du premier ordre semblent être la pauvreté, des conditions de vie horribles, la fragilisation des liens sociaux, l'insécurité objective, le sentiment d'insécurité, etc. (Beck, 1997; Chossudovsky, 1997; Bauman, 1998). Ces effets peuvent causer la mort et des attentes camouflées à la propriété et à la dignité humaine; bref, des coûts humains significatifs sans sujet direct et immédiat. Et par une sorte de causalité séquentielle et en boule de neige, on ne peut pas exclure la plausibilité d'effets de second ordre : l'émergence de comportements violents traditionnels ou autres formes de comportements problématiques et de résistance, y compris les incivilités, aisément pris en charge par le système pénal.

Certes, on doit se demander si l'on peut se référer aux «risques de la globalisation économique» (Beck, 1997) par l'expression «nouveaux dangers». Car il y a une sorte de symbiose entre les effets produits par la nouvelle politique de globalisation et ceux créés par la société industrielle. Mais il paraît y avoir un consensus quant à la nouveauté des mécanismes qui produisent «la globalisation de la pauvreté» (Chossudovsky, 1997). Ceux-ci ont pour effet de rendre de plus en plus difficile le contrôle des conséquences négatives par les moyens conventionnels. En effet, la nouvelle vague de concentration de la richesse est accompagnée par l'étouffement des institutions de l'État-nation et sa perte de contrôle en matière de politique économique. On observe aussi une neutralisation des mécanismes traditionnels de défense et de pacification sociale (syndicats, partis politiques, assistance sociale et fiscale, etc.). 
Chossudovsky (1997, p. 20) note que «dans l'Occident, le système démocratique a été placé devant un dilemme : les élus aux postes publics clés agissent chaque fois plus comme des bureaucrates et les créanciers de l'État deviennent les nouveaux dépositaires du pouvoir politique réel, agissant discrètement dans les arrière-scènes ». Beck (1997, p. 16) soutient que les chefs d'entreprise, surtout ceux qui se déplacent au niveau planétaire, ont réussi à reconquérir et à disposer du pouvoir de négociation qui avait été politiquement et socialement dompté dans le capitalisme organisé démocratiquement et peuvent exercer maintenant « un rôle clé non seulement dans la configuration de l'économie, mais de la société dans son ensemble». Le processus de perception de la dette externe, la réforme de la fiscalité et la position stratégique des entreprises transnationales font partie de ces transformations structurelles. Les grandes entreprises sont en mesure de séparer le lieu d'investissement, le lieu de production, le lieu de déclaration fiscale et le lieu de domicile (Beck, 1997, p.18). Au point où, selon André Gorz, «la majorité des compagnies multinationales, comme Siemens ou BMW, ne payent plus aucun impôt dans leur pays respectif» (cité par Beck, 1997, p. 21).

Les entrepreneurs, écrit Beck (1997, p. 18), «ont découvert la nouvelle formule magique de la richesse qui n'est autre que capitalisme sans travail plus capitalisme sans impôt ». La revue allemande Der Spiegel souhaite la bienvenue à cette politique : «Vive les profits, mort aux postes de travail!» (dans Beck, 1997, p. 20). Qu'il suffise de dire, en reprenant les remarques d'André Gorz (dans Beck, 1997, p. 21), que la croissance économique aux États-Unis n'a enrichi que les $10 \%$ plus fortunés de la population : ils ont reçu $96 \%$ de la richesse produite (ibid.). Et la situation en Allemagne est semblable : le profit des entreprises a augmenté de plus de $90 \%$ depuis 1979, alors que les salaires ont augmenté de $6 \%$. En plus, le fardeau fiscal relatif aux salaires a doublé dans les dix dernières années, et celui des activités des entreprises a chuté de la moitié, représentant seulement $13 \%$ de la recette fiscale globale, alors qu'en 1980 il représentait $25 \%$, et $35 \%$ en 1960 . Selon les estimations de Gorz, si l'on avait conservé le taux de $25 \%$, l'État allemand aurait eu dans les dernières années 80 millions de marks de plus par année. Il s'agit alors de nouveaux dangers décidés.

Or, la toile de fond du climat des années 1970 jusqu'à présent sera constituée, entre autres choses, par ces deux types de dangers décidés (industriels et socio-économiques). Je propose de répartir grosso modo l'observation de ce qui se passe par rapport à la question pénale sur deux axes, bien que l'on ne puisse pas les séparer intégralement. Le premier est celui des orientations sociales, préventives et de sécurité; le deuxième, celui des orientations législatives, judiciaires et correctionnelles. En général, la société du risque réactivera, sur ces deux axes, la rationalité pénale moderne (sous des formes anciennes et nouvelles). Cependant, pour comprendre les tensions et enjeux contemporains, il faut bien garder à l'esprit que cela ne va pas sans résistances et qu'il existe aussi, sur les deux axes, des modifications et des orientations innovatrices qui plongent leurs racines dans le climat d'auto-réflexion des années 1960 et 1970.

Disons aussi que l'impact de la société du risque sur ces deux axes fait encore l'objet de discussions et estimations dans plusieurs pays. Aux États-Unis, même s'il s'agit 
d'un pays de contrastes, la situation globale est claire : le système pénal et les politiques sociales et de sécurité ont pris rapidement des formes rétrogrades et inhumaines, allant d'une montée en flèche de l'incarcération et du retour à la peine de mort à une nouvelle orientation de la pénologie axée sur un objectif exclusif de neutralisation et de management des personnes criminalisées (Feeley et Simon,1992; 1994). Christie (1993) montre aussi la place que prend l'industrie en matière de contrôle strictement répressif poussant le système à s'en servir. En ce qui concerne d'autres pays, la situation semble aussi mauvaise, mais à une autre échelle et sous d'autres formes. En nous limitant ici aux études mettant l'accent sur l'axe des orientations sociales et de sécurité, Bauman (1998, p. 157) voit les États-nations investir massivement dans un mouvement d'ordre et sécurité sous «la forme d'une loi terrifiante et menaçante» qui atteint surtout, comme d'ordinaire, les classes les plus démunies de la société. Wacquant (1999, p. 11) attire l'attention sur l'émergence d'un «nouveau sens commun pénal visant à criminaliser la misère». Combessie (2001, p. 35-38) montre que plus de $60 \%$ des détenus en France métropolitaine n'ont pas un niveau d'éducation dépassant l'instruction primaire et sont en dessous du seuil de la pauvreté. Garland (1998, p. 51) y voit la naissance d'une «société punitive» où émerge un type de répression criminelle qui «n'est pas exclusivement lié à un régime politique». Mary (2001) voit dans les transformations récentes (surtout en Belgique) les signes du passage «d'un État social à un État (social) sécuritaire» qui réduit de plus en plus le sens du mot sûreté à la dimension répressive et qui fait appel à la peine comme une politique de réduction de risques ${ }^{18}$. Robert (1999, p. 259) ne voit pas des indicateurs d'une américanisation du contrôle pénal en Europe, mais trouve dans certaines fractions sociales une «hypersensibilité à l'insécurité» accompagnée d'une "forte exigence répressive» et signale que «l'enjeu consiste à distribuer de la sécurité [...] sans sombrer dans le sécuritaire». On voit donc se profiler à la fois l'abîme entre riches et pauvres, la détérioration de la qualité de la vie urbaine, la montée d'une insécurité diffuse et la "promotion spectaculaire des questions de “sécurité"»(Bauman 1998, p. 175).

\section{LA JURIDICISATION DE L'OPINION PUBLIQUE ET DU PUBLIC PAR LE SYSTÈME PÉNAL}

J'aimerais conceptualiser et attirer l'attention maintenant sur une transformation dans le système juridique contemporain que j'appelle la juridicisation de l'opinion publique et du public par le système (juridique) pénal. Elle peut être formulée comme suit. Le public commence à être observé, décrit et perçu par le système pénal comme étant ou devant être une composante de ce système et ce dernier réactive d'anciens mécanismes juridiques - ou organise de nouvelles formes — de participation normative du public qui vont bien au-delà du rôle strict, et fort bien encadré, du jury (ou encore du simple rôle de spectateur). Le public cesse d'être alors un simple destinataire de la norme

18. Pour une vue d'ensemble sur la situation en Belgique, voir aussi Van Campenhoudt, Cartuyvels, Digneffe, Kaminski, Mary et Rea, (2000) et Cartuyvels et Mary, (1999). Ces observations portent surtout, mais non exclusivement, sur l'axe des politiques sociales et de sécurité. 
juridique, ou encore un aspect de l'environnement du système pénal, pour devenir à la fois une sorte de critère et de prolongement interne de ce système.

Pour préciser la portée théorique de cette hypothèse, rappelons certaines réflexions faites par Luhmann. Il souligne que le processus de démocratisation déclenché au XVIII ${ }^{\mathrm{e}}$ siècle transforme le public en une composante du système politique. Le public émerge alors dans les sociétés modernes comme faisant partie du système politique et il assume à cet égard des rôles sociaux différents : payeur de taxes, partisan de résolutions, demandeur, électeur, écrivain de lettres aux éditorialistes, membre d'un groupe d'intérêt ou d'un mouvement social, etc. (Luhmann, 1982, p. 154). Il jouera ainsi un rôle croissant dans la production législative, en poussant le système politique à orienter - et à tenter de contrôler par ce moyen - le système juridique.

Notons en passant que pour Luhmann, la notion de public ne se confond pas avec l'ensemble des individus habitant un même territoire. En effet, le public se différencie du reste de la société "particulièrement en raison d'avoir accès seulement à des questions spécifiques et à des canaux de communication spécifiques» (Luhmann, 1982, p. 155). Cela veut dire que le public est toujours un public particulier constitué à partir de préoccupations ou d'enjeux spécifiques et, aussi, à partir des canaux (ou moyens) de communication spécifiques. La notion de public doit alors être envisagée en partie comme un rôle social que l'on prend ou que l'on construit, c'est-à-dire « une forme objectivée de participation de l'individu dans le processus social» (Dahrendorf, 1967, p. 41). Ainsi, un juge qui écrit une lettre au journal sur une question particulière fait partie du $p u-$ blic. Il s'agit d'un rôle que l'on prend dans certaines circonstances et chaque acteur peut assumer à différents moments des rôles différents (père de famille, juge, public, etc. $)^{19}$. Mais «public» est aussi une représentation sociale abstraite construite à partir de ces manifestations phénoménales diversifiées et atomisées ou encore à partir d'enquêtes et sondages d'opinion ${ }^{20}$.

Or, je crois que l'on peut dire qu'à partir des années 1960 et 1970, le public commence à être constitué aussi comme une composante de l'ensemble du système pénal par les opérations de reproduction de ce système $e^{21}$. Il est entendu que le système pénal intègre le public à sa façon et que cette intégration differe à certains égards de celle du système politique. Écartons un malentendu : le système pénal ne délègue pas le pouvoir de décision au public; c'est le système qui décide si, quand et comment le public sera intégré. Bien sûr, ceci suggère l'existence de nouveaux problèmes dans les rapports entre le système politique et le système juridique (pénal). Car, en un certain sens, ce processus produit une forme particulière de politisation du droit pénal et de la dogmatique pé-

19. Certes, il peut être important d'isoler parfois des constellations de rôles afin de distinguer un public profane d'un public expert et d'un public professionnel de la justice.

20. Voir à cet égard les réflexions classiques de Bourdieu (1973) à ce sujet.

21. Mon point d'ancrage théorique et empirique est le système pénal, mais comme une communication juridique de ce genre n'est pas étanche ou réservée à un sous-système, il y a lieu de croire que le public est devenu aussi une composante de l'ensemble du système juridique. Certaines remarques qui suivent suggèrent d'ailleurs cette extrapolation. Cependant, il y a assurément des problèmes qui sont propres au système pénal, et particulièrement celui relevant des peines. 
nale. Ainsi, par exemple, on peut se demander si le système politique et les tribunaux pénaux ne sont pas en train de devenir plus proches l'un de l'autre dans certains aspects du processus de prise de décisions et par quels moyens ou mécanismes ces rapprochements prennent place. Quoi qu'il en soit, dans le cadre de la justice pénale, cette transformation peut être (au moins en partie) mise en relation : i) avec l'expansion des médias et leur influence et impact en matière pénale; ii) avec l'importance accordée au public, aux sondages d'opinion publique et à la victimisation par le système politique et par les sciences sociales; iii) avec la participation croissante, au niveau du débat public, de mouvements sociaux — ou de segments de mouvements sociaux — «sans théorie ${ }^{22}$ sur le système pénal. Ces mouvements se caractérisent aussi par un engagement d'urgence dans des causes précises (par opposition à un engagement de réforme globale).

Disons aussi que cette intégration amène le public à participer différemment dans le processus de création et d'application de normes. Et l'intégration s'étend sur l'ensemble de l'espace juridique normatif : les normes (primaires) de comportements et les normes (secondaires) de sanctions, de procédure et concernant la justice correctionnelle. Cela veut dire que le système tiendra compte du public qu'il constitue autant dans la détermination de ce qui est licite ou illicite (normes primaires) que dans la détermination et gestion du remède légal au problème. Le système pénal modifie alors ses critères de décisions, son savoir sur les peines et s'engage dans une discussion pour déterminer les conditions de validité juridique de cette intégration, soit les nouvelles frontières du système pénal. Il crée aussi de nouveaux rôles et canaux de communication pour accueillir son public et construit à son tour la notion de public comme un personnage juridique anonyme et diffus avec lequel il tente de se mettre en communication de différentes manières. Au moins trois questions cruciales se posent : Une telle façon de procéder peut-elle permettre au système pénal de maintenir une façon autonome de concevoir la justice par rapport aux projets spécifiques du système politique et des mouvements sociaux ${ }^{23}$ ? Quelles peuvent être les conséquences de cette intégration si le système pénal ne modifie ni ses théories de la peine ni sa structure normative télescopée actuelle ( «qui fait $\mathrm{x}$ doit être puni par $\mathrm{y}$ )) ? Les décisions concernant le choix du procureur de recourir ou non aux tribunaux, le choix du type de chef d'accusation, le choix du type de procédure, le choix de la peine, le choix dans la détermination de ce qui est ou non une peine cruelle et inusitée, etc., doivent-elles dépendre du degré d'indignation du public, de la médiatisation du cas ou de l'envie du système pénal de communiquer avec son public?

Signalons aussi que Luhmann $(1974 ; 1996)$ avait déjà observé, par rapport au développement de la sémantique juridique en général au cours du $\mathrm{xx}^{\mathrm{e}}$ siècle, que celle-ci

22. C’est Luhmann (1986, p. 125) qui caractérise les nouveaux mouvements sociaux comme n'ayant pas de théorie. J'adapte cette remarque à mes propos car elle décrit très bien ce qui se passe ici. Voir aussi Feher et Heller (1984).

23. Cette première question s'inspire librement d'une question fondamentale, mais en général mal comprise, posée par Luhmann (1974, p. 31) vis-à-vis l'évolution de la dogmatique juridique dans son ensemble. 
avait commencé à prendre en compte l'intérêt de personnes et de groupes dans la qualité de «facteurs [ou critères] d'application de la justice»(Luhmann, 1996, p. 51) ${ }^{24}$. C'està-dire que les tribunaux, dans le cadre de leurs décisions, tiennent de plus en plus compte d'autres intérêts représentés dans le litige devant eux et aussi des conséquences qui peuvent découler de leur propre décision et qu'ils essayent de prévoir de façon erratique. Pour Luhmann, ce développement dans la dogmatique peut être attribué au fait que le droit constitutionnel (et le droit public en général) devient un moyen pour obtenir des avantages de l'État providence. On peut ajouter aussi que ce droit deviendra un moyen pour défendre des acquis dans la crise de l'État providence. À ma connaissance, les observations de Luhmann sur l'évolution de la dogmatique ne sont pas arrivées au point de l'amener à conceptualiser le public - qui pour lui était un sous-système du système politique — comme étant en train de devenir aussi une composante du système juridique (et moins encore du système pénal). Cependant, elles sont des indications empiriques sur ce qui se passe dans l'ensemble du système juridique et, à ce titre, contribuent à la formulation de l'hypothèse de travail proposée ici. Il faut cependant ajouter que dans le système pénal, en raison de sa sémantique propre et de la facilité par laquelle on glisse des normes de comportement aux normes sanctionnatrices, le public est construit aussi comme un critère de détermination et de gestion de la peine afflictive ${ }^{25}$.

Les signes de cette intégration-reconstruction du public par le système pénal sont multiples et exigent une recherche approfondie. Je me contenterai d'en indiquer trois, en privilégiant les tribunaux.

a. Tout d'abord, l'importance croissante accordée à l'idée de dénonciation tant au plan de la création des lois qu'au plan de la ratio de la sentence. La dénonciation sera

24. Luhmann (1974, p. 29) fait référence à des mouvements comme la jurisprudence d'intérêts, la jurisprudence sociologique, la méthode d'interprétation téléologique, l'approche d'ingénierie sociale, la pensée de prescriptions réalistes (realistisches Rezeptdenken), le mouvement d'usages alternatifs du droit, etc. Contrairement à ce que l'on prétend d'ordinaire à son égard, Luhmann n'est pas en train de soutenir la neutralité axiomatique du droit, mais plutôt d'attirer notre attention sur un problème. Celui-ci se caractérise par le fait que ces tendances n'ont pas élaboré encore une réflexion théorique susceptible d'auto-contrôler leur propre mouvement. Par conséquent, il se demande si cette tendance globale «peut être conciliée avec la fonction du droit et avec [sa] différenciation du système politique» (p. 30). Pour visualiser ce problème, je crois que l'on peut dresser un parallèle avec le système scientifique qui fait face à un problème analogue. La mise en cause de la prétendue neutralité axiologique de la science (qui est bienvenue), doit-elle nous conduire pour autant à la conclusion que la science doit produire ses connaissances comme le système politique? Comment tenir compte de la «non neutralité scientifique» et l'articuler avec la nécessité de ne pas transformer la production scientifique dans un simple projet politique? Etc.

25. Or, les notions de peine et de sanction ne sont pas conceptualisées de la même façon par les différents sous-systèmes juridiques. Dire que le droit s'appuie sur des sanctions ou punitions est trivial si l'on n'observe pas comment chaque sous-système théorise et rend opérationnelles leurs sanctions. Le droit civil, par exemple, se voit comme donnant des sanctions ou, au plus, des "peines imparfaites» parce qu'elles ont la victime comme bénéficiaire et ne valorisent pas directement la souffrance; le droit pénal, en revanche, s'auto-définit comme un droit de peines (par opposition à un droit de sanctions) et s'organise encore autour de l'incarcération. Dans ces conditions, le public du système pénal risque d'être surtout un public qui demande ce que le système lui promet ou enseigne de demander (Pires, Cellard, Pelletier, 2001). 
tantôt annexée à la théorie de la rétribution ou de la dissuasion, tantôt présentée comme une «nouvelle» théorie de la peine ou comme un nouvel objectif général des tribunaux (y compris du procureur de la couronne). Et même si certains jugements affirment heureusement que la dénonciation peut s'appliquer à une sentence qui n'implique pas l'emprisonnement, on voit s'établir quand même un lien entre la dénonciation et l'emprisonnement, soit-il sous la forme d'un sursis. Et le système établit aussi une équation entre l'intensité de la dénonciation (ou réprobation) et l'intensité de la répression. Les paroles du procureur et du juge sont dévalorisées en faveur de la violence de la peine. En effet, le système s'auto-décrit de plus en plus comme un média qui doit envoyer des «messages clairs, limpides et sans équivoques» à son public sur des sujets d'actualité, où «être clair» signifie «frapper fort».

En Angleterre, Cavadino et Dignan (1992, p. 40) signalent que le système pénal évoque le principe de la dénonciation dès le début des années 1950 dans le cadre d'une Commission Royale sur la peine capitale. Il est intéressant de voir comment il est construit en ayant le public comme critère et justification. En effet, Lord Denning fait la remarque suivante : «La punition pour des crimes graves doit adéquatement refléter la répulsion ressentie par la grande majorité des citoyens à leur égard. [...] L'ultime justification de la punition ce n'est pas la dissuasion, mais le fait d'être une dénonciation emphatique d'un crime par la communauté» (dans Cavadino et Dignan, 1992, p. 41).

Vers la fin des années 1970, le principe de la dénonciation s'entrelace de façon particulière avec les autres théories de la peine et gagne de l'importance dans le système, particulièrement pour justifier des peines (ou périodes) minimales d'incarcération ou encore des tarifs d'incarcération ${ }^{26}$. On songe même à guider l'opinion publique par la sévérité des peines ${ }^{27}$ et on laisse entendre que certaines décisions concernant la sévérité ont été prises en raison de la «forte publicité» entourant un fait divers ${ }^{28}$. Pour les tribunaux, la question ne semble plus être celle de savoir si la sévérité de la peine doit se construire à partir du public, mais plutôt celle de savoir si l'on doit et si l'on peut distinguer entre une sorte de préoccupation du public (public concern) à l'égard de certaines catégories générales d'offenses et l'indignation d'un public (public clamour) à l'égard d'un cas particulier, etc. Les tribunaux se lancent alors à la découverte du «sentiment public général» et considèrent cela une condition pour obtenir le «respect public pour l'administration de la justice». La sévérité de la peine semble, hélas, jouer un rôle central dans la conquête du respect. Enfin, nombre de propos expriment l'importance accordée par les tribunaux à l'apparence de justice. Ceci indique la présence de nouveaux intérêts ou influences et suscite une inquiétude : comment contrôler le dé-

26. C'est le cas de la Cour Suprême du Canada dans l'affaire Latimer (R. c. Latimer, 2001, CSC, parag. 86). 27. Voir, par exemple, cette autre décision d'une cour anglaise où Lord Justice Lawton dit : «la société, par la cour, doit montrer le fait qu'elle a horreur de certains types de crimes, et la seule façon par laquelle la cour peut montrer cela est à travers les peines (sentences) qu'elle octroie. Les tribunaux n'ont pas à refléter l'opinion publique. Mais d'autre part, ils ne doivent pas l'ignorer (must not disregard it). Peut-être le principal devoir de la cour est de guider l'opinion publique» (R. c. Sargeant, 1974, 60, Cr. App. Rep. 74, cité par Cavadino et Dignan 1992, p.56).

28. R. c. Latimer, 2001, Cour Suprême du Canada, parag. 86. 
rapage vers une justice répressive et erratique ou vers une justice préoccupée davantage par ce qui est extérieur au cas lui-même?29

b. L’Assemblée législative, les commissions de réforme et le système correctionnel vont considérer les consultations et les enquêtes d'opinion publique comme une sorte de baromètre, incluant même la réalisation de ces enquêtes dans leurs propres activités internes de travail. En outre, la victime et la police auront de plus en plus un autre rôle social : celui d'un public privilégié en termes de droit de consultation et de participation dans différentes étapes du système. Par exemple, on accordera aux victimes le droit d'assister aux exécutions dans les causes qui les concernent, de se prononcer au moment de la demande de libération conditionnelle sur l'impact que le délit continue à avoir sur leur vie (comme si cela était un élément nouveau depuis le procès et pouvait avoir une pertinence à l'égard de ce genre de décision $)^{30}$. Ces revendications, faites par certains mouvements de victimes, placent celles-ci dans le rôle social de public.

c. Depuis les années 1960, mais de façon plus intensive au Canada après l'adoption de la Charte des droits et libertés en 1982, on assiste à l'émergence massive de nouveaux acteurs sociaux dans les causes pénales devant les tribunaux ${ }^{31}$. Cela veut dire que l'on ne trouve pas seulement le représentant du ministère public contre l'inculpé, mais également d'autres avocats représentant des intérêts d'autres groupes dans le procès pénal. Aujourd'hui, dans une décision de la Cour Suprême du Canada, c'est un paysage familier que de voir une liste de mouvements sociaux qui sont venus à leur tour plaider dans le dossier. La fameuse formule juridique «Reine contre Untel» est-elle devenue obsolète? Doit-on désormais écrire «Reine plus tel ou tel groupe ou organisation contre Untel $»^{32}$ ? Les effets apparents de ce miracle de la multiplication des pains semblent être, au moins dans certains cas, la dispersion d'intérêts et de raisons de toutes sortes pour faire décider en faveur ou contre le plaignant ${ }^{33}$ et l'exacerbation du déséquilibre dans les rapports de force entre l'accusation et la défense pour un bon nombre d'inculpés. Comme la grande majorité des inculpés continue à venir des couches moins favorisées et n'a pas d'accès égal aux avocats, on peut s'inquiéter de cette forme de démocratisation du système.

29. Cette préoccupation s'exprime au plan judiciaire, mais aussi législatif. Voir Erhel (1993), Garapon (1993), Jodouin (2000).

30. Voir le rapport du Sous-comité sur la Loi sur le système correctionnel du Comité permanent de la justice et des droits de la personne de la Chambre des communes du Canada (mai 2000, chapitre 8).

31. Plusieurs études ont attiré l'attention sur le phénomène de la multiplication des acteurs juridiques dans l'ensemble du système juridique, mais je ne connais pas de recherches essayant de montrer les conséquences de ce phénomène spécifiquement en matière pénale ou essayant d'évaluer le nouveau rapport de force que cela est susceptible de créer.

32. Bien entendu, dans certains cas, que je suppose pour l'instant plus rares au pénal, certains groupes s'alignent aussi du côté de l'Untel, contre la Reine. Il faut alors estimer, empiriquement, le poids des groupes qui s'opposent. Nous envisageons présentement une recherche sur cette question.

33. La pratique de "pondération d'intérêts divergents» en droit pénal (Jodouin, 1996, p. 807), surtout lorsqu'il s'agit de questions relevant de la dignité humaine, peut faire dévier complètement l'enjeu central du jugement. 
Au Canada, la participation des parties au procès se fait, entre autres choses, par la réactivation d'un ancien mécanisme du common law : la figure de l'amicus curiae ( «l'ami de la cour»). Dans le passé, il s'agissait d'une personne qui donnait une information à la cour sur un point en litige ou encore d'une personne qui n'avait pas en principe le droit d'être partie dans une cause, mais qui était autorisée à venir défendre ses propres intérêts. Il semble que ce mécanisme n'était pas intensivement déployé au pénal et que c'est bien l'activation des droits de la personne qui a ouvert grandes les portes à son utilisation.

Pour différentes raisons, la justification de la sévérité de la peine par le système pénal en fonction directe du public, de l'état d'esprit de la communauté, de la clarté du message, de la médiatisation du cas, etc., est entièrement étrange à la façon de penser de la grande majorité des philosophes et juristes des XVIII et ${ }^{\mathrm{e}} \mathrm{XX}^{\mathrm{e}}$ siècles. Une panoplie de raisons ont été mises à contribution, mais la sévérité de la peine demeurait un calcul utilitariste savant, le fruit d'un raisonnement moral ou une question de droit exigeant dans tous les cas, tant de la part du législateur que du juge, un «élément froid» (Simmel) dans la chaleur de l'enjeu . Néanmoins, il y a eu au moins une exception remarquable. En effet, Gabriel Tarde (1890, p. 507 et suiv.) a anticipé la place de l'opinion publique dans une théorie de la peine. Il soutient que le but de cette dernière ne devrait pas s'appuyer sur une quelconque utilité (ou théorie rétributiviste), mais plutôt sur une «volonté générale» conçue comme opinion. Mais Tarde remplace le concept éthique et politique conventionnel de concept de volonté générale par l'idée d'intérêt public, intérêt $d u$ public, opinion $d u$ public (par rapport à un problème général). Il dira alors que la prétention d'être utile au peuple en dépit de sa volonté «s'appelle, en politique, absolutisme; en droit pénal cela s'appelle utilitarisme». De façon lapidaire, il écrira : «Assise sur l'opinion, la peine me paraît tout autrement justifiable qu'assise sur l'utilité» (p. 509). Bref, une nouvelle façon d'ancrer dans le sable mouvant la notion creuse de proportionnalité. Il rejoint alors la tendance contemporaine à transformer le public en une sorte de «source du droit de punir» non identifiée comme telle.

Bien entendu, on ne peut pas dire que l'intégration du public comme une composante de la justice pénale produise nécessairement et dans toutes les circonstances des effets pervers. Ceci est d'ailleurs une question empirique, car elle dépend en bonne partie de comment le droit pénal s'organise lors de cette intégration. Mais pour que l'intégration du «public» puisse s'accomplir de façon innovatrice, elle doit être accompagnée d'un mode de pensée alternatif, tant sur le plan cognitif que de l'auto-organisation normative du système, ce qui est un phénomène aussi souhaitable que rare. Cependant, comme le droit pénal n’a transformé substantiellement ni sa structure normative ni sa rationalité, nous pouvons au moins montrer un aspect de cette intégration qui constitue un embarras pour la démocratie contemporaine : les demandes de pénalisation accrue fondées sur le droit de la personne.

En effet, on distingue d'ordinaire au moins deux catégories de droits de la personne : les droits-barrières» (droits de...) et droits-créances (droit à...) (Mourgeon, 1978; Loschack, 1984). Les premiers visent à garantir une zone d'autonomie indivi- 
duelle, limitant l'ingérence de l'État (et des particuliers) dans la vie (privée) des individus. Les seconds sont les «droits à l'obtention» (Mourgeon) et visent à promouvoir le bien-être social de l'acteur qui le revendique ${ }^{34}$. Or, le système pénal invente, avec l'intégration du public, une curieuse sorte de droit-créance, un droit-créance négatif: le droit à la souffrance des inculpés comme une forme d'auto-estime, d'estime des valeurs ou de protection imaginaire. La conception selon laquelle la peine doit être un tarif, jumelée à la valorisation de la personne humaine et à celle d'une égalité d'effet et de nature concernant la peine vont créer un nouveau dicton cartésien, adapté à la rationalité pénale : «dites-moi quelle peine afflictive vous donnez, et je saurai combien je vaux » ${ }^{35}$. À ne pas se tromper, cette représentation est construite et enseignée par la rationalité du système. Pour reprendre les termes de Sack (1968, p. 469), disons que cette demande et ce droit à la souffrance sont la contrepartie du bien négatif (negatives Gut) — le stigmate et la souffrance - distribué et promis par le système pénal. Dans ce cadre, la souffrance demandée ou à imposer n'est jamais suffisante pour compenser les pertes ou prévenir les risques.

Mais quel est l'aspect particulier de ce droit à la souffrance des inculpés? Comparons quatre types de demandes distinctes en matière pénale. Dans la demande pour créer un nouveau crime, l'acteur cherche à généraliser ou à honorer une expectative normative de comportement qui lui tient à cœur. Cette criminalisation lui apporte un gain juridique, soit la consécration légale d'une expectative de comportement. En sens inverse, la demande de décriminalisation (par exemple, de l'avortement, etc.) est aussi un gain juridique : l'acteur revendique au moins un espace de liberté ou le droit-barrière de ne pas subir une conséquence juridique particulière. Comme le dirait Dahrendorf (1967, p. 61), on veut transformer ici une expectative normative rendue obligatoire par les lois en une expectative préférentielle ou permissive. Par rapport aux peines afflictives, le problème devient un peu plus complexe. Il reste que la demande pour dépénaliser, c'est-à-dire pour créer des sanctions alternatives, abolir les peines minimales, réduire la durée d’une peine, etc., s'appuie sur des valeurs positives ou des vertus ${ }^{36}$. Cette revendication produit aussi une sorte de gain moral ou juridique. Par ailleurs, il y a une asymétrie entre toutes ces demandes et celle pour aggraver la sévérité d’une peine déjà afflictive (pénalisation

34. Selon une autre classification, on distinguera trois sortes de droits de la personne : les droits politiques, les droits-protection et les droits-créances. Les droits politiques auraient alors la fonction normative d'habiliter l'individu à certains droits ou comportements. Mais cette classification n'affecte pas mes propos.

35. Ce message a été reçu par le public et circule régulièrement dans le système. Le représentant d'un groupe de pression, qui était présent à la Cour Suprême du Canada lors d'un de ses jugements, déclare : «Si Robert Latimer [condamné pour avoir tué par amour et compassion sa fille handicapée] obtient une sentence de deux ans [au lieu d'une peine de prison à perpétuité sans possibilité de libération conditionnelle avant 10 ans], cela enverra le message que les personnes handicapées ne sont que le cinquième d'un être humain». Comme on le voit, la période de dix ans pour la demande de la libération conditionnelle indique maintenant la valeur d'un être humain et la peine doit exprimer cette valeur.

36. Il y a une exception. Il arrive souvent en droit pénal que la simple demande pour réduire le quantum d'une peine afflictive soit motivée par le désir de produire la souffrance. Cela arrive lorsque l'acteur est convaincu que l'impunité pénale d'un comportement est due à la sévérité excessive d'une peine prévue par la loi (Pires, Cellard, Pelletier, 2001). 
accrue $)^{37}$. Dans ce dernier cas, la demande est, pour ainsi dire, «vide de gains». D'où l'embarras pour les démocraties contemporaines : l'invention d'un simple droit à l'antibien-être social des autres, une catégorie paradoxale de droits de la personne : un droit purement contre la personne, sans gain juridique ou moral concret et immédiat autre que celui de produire la souffrance. C'est un droit qui ne reconnaît pas l'existence d'un nous — d'une appartenance (belong) — qui inclut le déviant (Mead, 1918, p. 586).

\section{RÉSUMÉ}

Cette étude décrit certaines caractéristiques de la rationalité pénale moderne comme un système de pensée autonome et explore quelques aspects de l'interaction entre les systèmes économique, politique et juridico-pénal dans les sociétés contemporaines («société du risque»). Entre autres choses, il attire l'attention sur les rapports contradictoires entre les droits de la personne et le droit pénal et conceptualise un phénomène nouveau qui émerge dans la deuxième moitié du XX siècle : la juridicisation de l'opinion publique et du public par le système pénal. En effet, le public devient une composante du système pénal et cela produit un rapprochement problématique entre le système politique, les projets de toute sorte de mouvements sociaux et le système pénal. On pourra alors mieux voir certains embarras que posent la rationalité et la structure normative du droit pénal moderne pour les démocraties contemporaines.

\section{SUM MARY}

This study describes certain characteristics of modern penal rationality as a system of autonomous thought and explores some aspects of the interaction between economic, political and legalcriminal systems in contemporary societies ("societies at risk"). It calls attention, among other things, to the contradictory relationships between individual rights and the penal system and conceptualizes a new phenomenon that is emerging in the second half of the $20^{\text {th }}$ century: the legalization of public opinion and of the public by the penal system. In fact, the public is becoming a component of the penal system, and this is bringing about a problematical rapprochement between the political system, social movement projects of all kinds, and the penal system. Certain difficulties that rationality and the normative structure of modern criminal law have posed for contemporary democracies can be better appreciated from this perspective.

\section{RESUMEN}

Este estudio describe ciertas características de la racionalidad penal moderna como un sistema de pensamiento autónomo y explora algunos aspectos de la interacción entre los sistemas económico, político y jurídico-penal en las sociedades contemporáneas («sociedad de riesgo»). Entre otras cosas, se llama la atención sobre las relaciones contradictorias entre los derechos de la persona y el derecho penal, y se conceptualiza une nuevo fenómeno que emerge en la segunda mitad del siglo XX: la juridización de la opinión pública y del público por el sistema penal. En efecto, el público se vuelve una componente del sistema penal y eso produce un acercamiento problemático entre el sistema político, los proyectos de toda clase de movimientos sociales y el sistema penal. Se podrán entonces ver mejor ciertos problemas que provocan la racionalidad y la estructure normativa del derecho penal moderno para las democracias contemporáneas.

37. Il ne faut pas confondre cette situation avec celle où l'on demande l'intervention du système pour soutenir certaines expectatives là où il fait très peu ou rien. Dans ces cas, on demande que le système donne des sanctions ayant une certaine signification, mais il n'y a pas de rapport fixe établi entre la valeur d'un bien protégé et le type ou le quantum de la sanction. 


\section{BIBLIOGRAPHIE}

Acosta, F. (1987), «De l'événement à l'infraction : le processus de mise en forme pénale», Déviance et Société, vol. 11, no 1, p. 1-40.

Bauman, Z. (1998), Le coût humain de la mondialisation, Paris, Hachette, 1999.

Beck, U. (1997), Qué es la Globalización? Falacias del globalismo, respuestas a la globalización, Barcelona, Ediciones Paidós Ibérica, 1998.

Beck, U. (1994), «D’une théorie critique de la société vers la théorie d'une auto-critique sociale», Déviance et Société, vol. 18, nº 3, p. 333-343.

BeCK, U. (1993), «De la société industrielle à la société à risques. Problématiques de la survie, structures sociales et éveil d'une conscience écologique», Revue Suisse de Sociologie, vol. 19, p. 311-337.

Beck, U. (1986), Risk Society. Towards a New Modernity, Londre, Sage Publications, 1992.

Bцoch, E. (1961), Droit naturel et dignité humaine, Paris, Payot, 1976.

Воввіо, N. (1961), O Positivismo Jurídico. Lições de Filosofia do Direito, São Paulo, Icone, 1999.

Bourdieu, P. (1993), «Esprits d'État. Genèse du champ bureaucratique», Actes de la recherche en sciences sociales, vol. 96-97, p. 49-62.

Bourdieu, P. (1973), «L'opinion publique n'existe pas», Les Temps Modernes, vol. 29, nº 318, p. 1292-1309.

Carbonnier, J. (1972), Sociologie juridique, Paris, Armand Colin.

Cartuyvels, Y. et Ph. Mary (dir.) (1999), L'État face à l'insécurité. Dérives politiques des années 90, Bruxelles, Labor.

Cavadino, M. et J. Dignan (1992), The Penal System, Londres, Sage.

Chossudovsкy, M. (1997), The Globalisation of Poverty — Impacts of IMF and World Bank Reforms, Penang, Third World Network.

Christie, N. (1993), Crime Control as Industry: towards Gulag, Western Style?, Londres, Routledge.

Combessie, P. (2001), Sociologie de la prison, Paris, Éditions La Découverte.

DAhrendorf, R. (1967), Homo Sociologicus. Ensaio sôbre a história, o significado e a crítica da categoria de papel social, Rio de Janeiro, Tempo Brasileiro, 1969.

Del Vecchio, G. (1955), La justice — La vérité. Essai de philosophie et morale, Paris, Dalloz.

Durkheim, É. (1969), «Devoirs généraux indépendants de tout groupement social. L'homicide», in É. Durkheim, Leçons de sociologie, Paris, Puf, p. 142-151.

Durkheim, É. (1963), L'éducation morale, Paris, puf.

Durкheim, É. (1895), «Crime et santé sociale», Revue philosophique de la France et de l'étranger, vol. 39, p. $518-523$.

Ehrlich, E. (1986), Fundamentos da Sociologia do Direito, Brasília, Éditions de l’Université de Brasilia.

Erhel, C. (1993), «La loi et le fait-divers», Esprit, vol. 195 (octobre), p. 103-109.

Feeley, M. et J. Simon (1994), «Actuarial Justice: The Emerging New Criminal Law», in D. Nelken (dir.), The Futures of Criminology, Londres, Sage, p. 193-201.

Feeley, M. et J. Simon (1992), «The New penology: Notes on the Emerging Strategy of Corrections and Its Implications», Criminology, vol. 30, no 4, p. 449-474.

Feher, F. et A. Heller (1984), «From Red to Green», Telos, vol. 52, p. 35-44.

GARAPON, A. (1993), «Le législateur irrationnel», Esprit, vol. 195 (octobre), p. 109-117.

GARLAND, D. (1998), «Les contradictions de la société punitive : le cas britanique», Actes de la recherche en sciences sociales, vol. 124, p. 49-67.

GavazzI, G. (1967), Norme primarie e norme secondarie, Torino, G. Giappichelli, Memorie dell'Instituto giuridico, Università di Torino.

Giddens, A. (1990), Les conséquences de la modernité, Paris, Harmattan, 1994.

Grotius, H. D. G. (1625), Le droit de la guerre et de la paix, Paris, Guillaumin, 1867.

Hart, H. L. A. (1961), Le concept de droit, Bruxelles, Publications des Facultés universitaires Saint-Louis, 1988. Jodouin, A. (2000), «La légitimité des sources du droit pénal. Réflexions d'un agnostique sur les certitudes fondamentales du droit répressif», in E. MACKAAY (dir.), Les certitudes du droit, Montréal, Thémis, p. 115-150. 
Jodouin, A. (1996), «La charte canadienne et la nouvelle légalité», in G.-A. BeAudouin et E. P. Mendes (dir.), Charte canadienne des droits et libertés, Montréal, Wilson \& Lafleur, p. 789-820.

Kant, E. (1785), Fondements de la métaphysique des mours, Paris, Delagrave, 1991.

Kelsen, H. (1979), Théorie générale des normes, Paris, puf, 1996.

Lazarou, J., B. H. Pomeranz et P. N. Corey (1998), «Incidence of Adverse Drug Reactions in Hospitalized

Patients. A Meta-analysis of Prospective Studies ", Journal of the American Medical Association, vol. 279, (Avril, 15), p. 1200-1205.

Loschaк, D. (1984), «Mutation des droits de l'homme et mutation du droit», Revue interdisciplinaire d'études juridiques, vol. 13, p. 49-88.

Luhmann, N. (1997), Das Recht der Gesellschaft, Frankfurt, Suhrkamp.

Luhmann, N. (1996), Introducción a la teoría de Sistemas. Lecciones publicadas por Javier Torres Nafarrate, Barcelone, Anthropos; Méxique, Universidad Iberoamericana y Iteso.

Luhmann, N. (1993), Risk: A sociological theory, New York, Aldine De Gruyter.

Luhmann, N. (1986), Ecological Communication, Chicago, University of Chicago Press, 1989.

Luhmann, N. (1982), «Politics as a Social System», in N. Luhmann, The Differentiation of Society, Columbia, Columbia University Press, p. 138-165.

Luhmann, N. (1979), Trust and Power: Two works, Toronto, Viley.

Luhmann, N. (1974), Rechtssystem und Rechtsdogmatik, Stuttgart, Verlag W. Kohlhammer GmbH.

Mary, Рh. (2001), «Pénalité et gestion des risques : vers une justice "actuarielle” en Europe ?», Déviance et Société, vol. 25, n 1, p. 33-51.

Mead, G. H. (1918), "The Psychology of Punitive Justice», American Journal of Sociology, vol. 23, n 5 , p. 577-602.

Mourgeon, J. (1978), Les droits de l'homme, Paris, Puf.

Muller, J.-M. (1995), Le principe de non-violence. Parcours philosophique, Paris, Desclée de Brouwer.

Pires, A. P. (2001a), «La ligne Maginot en droit pénal : la protection contre le crime versus la protection contre le Prince», Revue de droit pénal et de criminologie, vol. 81, nº 2, p. 145-170.

Pires, A. P. (2001b), «Consideraciones preliminares para una teoría del crimen como objeto paradojal», in M. Sozzo (dir.), Futuros Fragmentados. Visiones desde / sobre las criminologías contemporaneas, Buenos Aires, AD-HOC (à paraître).

Pires, A. P. (1998a), «Aspects, traces et parcours de la rationalité pénale moderne», in C. Debuyst, F. Digneffe et A. P. Pires (dir.), Les savoirs sur le crime et la peine. La rationalité pénale et la naissance de la criminologie, tome 2, Bruxelles, De Boeck Université, p. 3-52.

Pires, A. P. (1998b), «Kant face à la justice criminelle», in C. Debuyst, F. Digneffe et A. P. Pires, Les savoirs sur le crime et la peine. La rationalité pénale et la naissance de la criminologie, tome 2, Bruxelles, De Boeck Université, p. 145-206.

Pires, A. P. (1998c), «Un noud gordien autour du droit de punir», in C. Debuyst, F. Digneffe et A. P. Pires, Les savoirs sur le crime et la peine. La rationalité pénale et la naissance de la criminologie, tome 2, Bruxelles, De Boeck Université, p. 207-220.

Pires, A. P., A. Cellard et G. Pélletier (2001), «L'énigme des demandes de modifications législatives au Code criminel canadien», in P. Fraile (dir.), Modelar para gobernar. El control de la población y el territorio en Europa y Canada, Barcelone, Éditions de l' Université de Barcelone, p. 207-231.

Robert, Ph. (1999), Le citoyen, le crime et l'État, Paris, Droz.

SACK, F. (1968), «Neue Perspektiven in der Kriminologie», in F. SACK et R. KöNIG (dir.), Kriminalsoziologie, Frankfurt, Akademische Verlagsgesellschaft, p. 431-475.

TARDE, G. (1890), La philosophie pénale, Paris, Cujas, 1972.

van Campenhoudt, L., Y. Cartuyvels, F. Digeffe, D. Kaminski, Ph. Mary et A. Rea (dir.) (2000), Réponses à l'insécurité. Des discours aux pratiques, Bruxelles, Labor.

von Feuerbach, A. R. (1801), Lehrbuch des gemeinen in Deutschland gültigen peinlichen Rechts, Giesen, G. F. Heyer Verlag, 1847.

WACQuAnt, L. (1999), Les prisons de la misère, Paris, Éditions Raisons d'agir. 